\title{
A Clinical Laboratory Paradigm for Evaluating Medication Effects on Alcohol Consumption: Naltrexone and Nalmefene
}

\author{
David J Drobes*,', Raymond F Anton', Suzanne E Thomas' and Konstantin Voronin' \\ 'Charleston Alcohol Research Center, Department of Psychiatry and Behavioral Sciences, Medical University of South Carolina, USA
}

\begin{abstract}
Opiate antagonist medications have been shown to improve alcoholism treatment, but few human laboratory-based studies investigating mechanisms for these effects have been conducted on alcohol dependent persons. The present study was designed to determine the impact of two opiate antagonists on alcohol consumption among nontreatment-seeking alcoholics $(n=125)$ and social drinkers $(n=90)$. Participants were randomly assigned to receive placebo, naltrexone (titrated to $50 \mathrm{mg} /$ day), or nalmefene (titrated to $40 \mathrm{mg} /$ day) for 8 days with an alcohol laboratory session on the final day. Alcohol consumption was monitored in the natural environment during the first 5 medication days, and during a choice consumption paradigm following a standard 'priming' alcohol dose in a bar-laboratory setting. Social drinkers consumed less alcohol than alcoholics during the prelab medication period and the laboratory choice consumption paradigm, and they attained lower blood alcohol levels than alcoholics following the priming drink. Both opiate antagonist medications equally reduced drinking amounts and frequency among alcoholics but not social drinkers, relative to placebo, during natural environment and bar-lab alcohol consumption evaluations. Greater medication side effects, mostly mild in nature, were observed in participants taking nalmefene. These findings demonstrate that both naltrexone and nalmefene can lead to reductions in alcohol consumption among alcoholics who are not attempting to reduce drinking. Similar laboratory paradigms may offer substantial advantages for observing these effects during evaluation of other medications as well.

Neuropsychopharmacology (2003) 28, 755-764. doi: I 0.1038/sj.npp. I 300101
\end{abstract}

Keywords: alcohol; social drinkers; naltrexone; nalmefene; opiate antagonists; laboratory

\section{INTRODUCTION}

Over the past decade, randomized placebo-controlled clinical trials have shown opiate antagonist medications to be effective in the treatment of alcoholism. In particular, naltrexone reduces relapse risk during and following treatment and is associated with other improved drinking outcomes (Anton et al, 1999; O'Malley et al, 1992, 1995; Volpicelli et al, 1992, 1997). Another opiate antagonist medication, nalmefene, has also shown beneficial treatment effects (Mason et al, 1994, 1999). Despite these promising findings, the observed effect sizes with opiate antagonist agents have been rather modest and somewhat variable (Kranzler and Van Kirk, 2001). Not surprisingly, positive outcomes appear to depend largely on treatment compliance (eg Chick et al, 2000; Volpicelli et al, 1997). In addition, a recent multisite controlled trial with chronic, severe alcohol-dependent men did not find that naltrexone

\footnotetext{
* Correspondence: Dr DJ Drobes, Tobacco Research and Intervention Program, H. Lee Moffitt Cancer Center \& Research Institute, University of South Florida, 4I I 5 E. Fowler Ave., Tampa, FL 336 I7, USA, Tel: 8I 3 632- I75I, Fax: 813-632- 1755, E-mail: drobesdj@moffitt.usf.edu Received 10 January 2002; revised 17 April 2002; accepted 25 October 2002

Online publication: 30 October 2002 at http://www.acnp.org/citations/ Npp 103002419
}

improved drinking outcomes (Krystal et al, 2001). More information is clearly needed to better understand how opiate antagonists influence drinking and how to optimize their clinical use and foster improved formulations.

Clinical laboratory paradigms can provide time and costefficient methods for exploring mechanisms underlying medication effects using carefully controlled conditions. For instance, laboratory paradigms can be used to determine the effects of opiate antagonists on alcohol consumption without the time and expense of a major treatment outcome study, perhaps leading to insights that would improve the clinical usage of these agents. In addition, laboratory models can be used to evaluate novel agents and compare their effects with more established medications. The primary goal of the present study was to utilize a clinical laboratory paradigm for evaluating opiate antagonist effects on alcohol consumption in both natural environment and laboratory settings.

Several recent laboratory-based studies have examined effects of naltrexone on alcohol consumption and alcohol response (eg Davidson et al, 1996, 1999; de Wit et al, 1999; King et al, 1997a; McCaul et al, 2000; O'Malley et al, 2002; Palfai et al, 1999; Swift et al, 1994). In several of these studies, naltrexone has been shown to attenuate alcohol consumption in drinkers who consume various amounts of alcohol. However, this effect has not always been observed 
(eg Palfai et al, 1999) and some findings have suggested that drinking reductions may not be specific to alcohol consumption, per se (de Wit et al, 1999). Recent findings suggest that previously observed naltrexone-induced reductions in stimulatory effects of alcohol (eg Swift et al, 1994) may be evident only in subjects at high risk for alcohol dependence (King et al, 1997a) or those with high positive alcohol outcome expectancies (Palfai et al, 1999).

Most of the laboratory studies described above were conducted on social drinkers. Accordingly, it is unclear to what extent findings from these studies are generalizable to alcoholics - those who are most likely to receive opiate antagonist treatment. Furthermore, most studies have employed acute medication dosing regimens (ie single dose) that bear little similarity to parameters that are likely to be utilized in treatment settings. One recent laboratory study examining naltrexone effects on alcohol consumption and response was conducted on nontreatment-seeking alcoholics (O'Malley et al, 2002). This placebo-controlled study used subacute (6 days) dosing, and included an extensive array of measures to characterize subjective and hormonal response following a standard alcohol priming drink, as well as alcohol consumption during a subsequent alcohol self-administration test. These researchers found that naltrexone was associated with reduced alcohol craving, as well as attenuated alcohol consumption, during the laboratory test session. Interestingly, reductions in alcohol craving were associated with activation of the hypothalamus-pituitary-adrenocortical (HPA) axis (ie cortisol). Although based on a small sample size, this preliminary study raises some provocative hypotheses regarding potential mechanisms underlying naltrexone effects in the treatment of alcoholism.

The present laboratory study was designed to evaluate opiate antagonist effects on alcohol consumption as a function of drinking status. Therefore, we studied separate groups of nontreatment-seeking alcoholics and social drinkers, matched according to age and gender. In addition to inclusion of a comparison group of social drinkers, another departure from the O'Malley et al (2002) study is that the present alcohol self-administration procedure was conducted in a controlled bar-like setting and involved consumption of a preferred alcoholic beverage. A 1-week medication regimen was employed prior to the consumption laboratory session, with no restrictions on drinking during the first 5 days of the medication period. All alcohol administration procedures were conducted in accordance with the guidelines espoused by the National Advisory Council of the National Institute on Alcohol Abuse and Alcoholism (NAC Guidelines, 1988) and all procedures were approved by the University's IRB.

There have been few attempts to directly compare medications for alcoholism treatment, with the vast majority of studies focusing on one drug in comparison to placebo. An impediment to comparing two or more medications is that treatment studies of this type are lengthy and expensive to conduct. In addition to the primary study goal of evaluating opiate antagonist effects on alcohol consumption in alcoholics and social drinkers, we directly compared the effects of naltrexone and nalmefene on drinking behavior in both natural environment and barlab settings. The primary differences between these two opiate antagonist agents are that nalmefene has a longer half-life and a somewhat different pharmacological profile (eg De Haven-Hudkins et al, 1990; Emmerson et al, 1994; Mitch et al, 1993; Wang et al, 2001). Based on these differences, it was hypothesized that nalmefene might reduce drinking more than naltrexone.

\section{METHOD}

\section{Participants}

A total of 125 alcoholics and 90 social drinkers between the ages of 21 and 65 years participated in this study. Potential participants, recruited through newspaper and community ads, were told that the study was investigating effects of two medications, both previously shown to have beneficial effects for alcoholics in treatment, on reactions to alcohol in a laboratory situation. They were not given any specific details about possible medication effects on craving, alcohol consumption, or effects of alcohol. Following telephone screening, potential participants who appeared eligible were invited to attend a detailed evaluation session. All alcoholic participants met DSM-IV criteria for alcohol dependence (American Psychiatric Association, 1994), including loss of control drinking or an inability to cut down or quit, but they denied any active involvement in, or desire for, alcohol treatment. Social drinkers had no history of alcohol abuse or dependence, and currently reported drinking no more than 14 standard drinks per week, no more than five drinks per occasion, but at least two drinks on one occasion during the previous 30 days. This final criteria ensured that no participant was given more alcohol as a 'priming' drink during the bar-lab procedure than he/she had consumed on a single occasion in the recent past. Exclusion criteria for all participants were as follows: current DSM-IV criteria for drug dependence (excluding nicotine) by verbal report and urine drug screens, other major DSM-IV Axis I disorders, past opiate abuse or dependence, psychoactive medication or substance use (except marijuana) in the past 30 days, current suicidal or homicidal ideation, past history of alcohol-related medical illness, liver enzymes $\geqslant 2.5$ times above normal, or significant health problems.

\section{Procedures}

Upon arrival for the first session, the study was described in detail to the participant and Informed Consent was obtained using a form and procedures approved by the IRB at our institution. Each participant was then evaluated with a number of standard interview, questionnaire, and medical diagnostic procedures. Interview procedures included a demographic form, the alcohol and drug section of the Structured Clinical Interview for the DSM (SCID; First et al, 1994), a timeline follow-back interview to quantify drinking during the preceding 90 days (Sobell and Sobell, 1992), and a structured Family History Research Diagnostic Interview (Andreasen et al, 1977) to obtain information on alcohol and other substance use problems among first- and second-degree relatives. Self-report measures administered during the first session included the Obsessive-Compulsive Drinking Scale (OCDS; Anton et al, 1995), the SelfAdministered Alcohol Screening Test (SAAST; Davis et al, 
1987), the Alcohol Dependence Scale (ADS; Skinner and Allen, 1982), the Beck Depression Inventory (BDI; Beck, 1967), and the Beck Anxiety Inventory (BAI; Beck et al, 1988). Finally, a urine specimen was collected to screen for abused drugs, and a blood sample collected for liver function and general health screening. Additional assessments were conducted at a second session (conducted within 1 week of the first session), including psychiatric sections of the SCID, the Alcohol Craving Questionnaire (ACQ; Singleton et al, 1999), and a baseline symptom checklist (to compare with later medication side effects). In addition, a physical exam was conducted by a physician assistant.

Participants who passed all screening and eligibility criteria were assigned to receive naltrexone, nalmefene, or placebo using an urn randomization procedure (eg Stout et al, 1994). This procedure was designed to balance medication groups according to gender, smoking status, family history of alcohol problems, and preferred beverage (beer, wine, or liquor). The number of participants who were assigned to each medication condition were as follows: Social Drinkers (placebo, 30, naltrexone, 29, nalmefene, 31); Alcoholics (placebo, 50, naltrexone, 39, nalmefene, 36). The medication regimen was for 8 days, and consisted of the following dose titration for naltrexone: $25,25,50,50,50,50$, $50,50 \mathrm{mg} /$ day. The first seven participants who were assigned to receive nalmefene received the following titration: 20, 20, 40,40,40,40,40, 40 mg/day. However, because of intolerable side effects (eg dysphoria, temperature dysregulation, cognitive disturbances, illusions, sleep difficulties) reported by three of these initial participants (two in the social drinker group, one in the alcoholic group), the titration was slowed to the following for the remainder of the study participants: $5,10,20,40,40,40,40$, $40 \mathrm{mg} /$ day. All medications, including inactive placebo, were administered in standard gelcaps with $100 \mathrm{mg}$ riboflavin added to assess for compliance via flourescence assay (see Sullivan et al, 1989a).

Participants were given no explicit instructions regarding use of alcohol or modification of their drinking behavior for days 1-5. However, they were required to abstain completely from drinking on days 6 and 7. Participants were clinically evaluated for alcohol withdrawal in person on each of these 2 days using the Clinical Institute Withdrawal Assessment for Alcohol-Revised (CIWA-ar; Sullivan et al, 1989b), and a urine sample was collected to ascertain medication compliance. On day 6, participants were also administered a 5-day version of the timeline follow-back interview, in which they reported their alcohol consumption since the outset of the medication period. In addition, medication ingestion was witnessed on days 1,6 , and 7. On day 7, participants were familiarized with the bar-lab room in which the alcohol consumption assessment would be conducted the following day. However, alcoholspecific cues (bar, barstools, alcohol bottles on shelves, signs, etc) were shielded from the participants' view by a curtain.

On day 8, participants attended a session in the bar-lab setting, including a standard alcohol administration and an alcohol choice self-administration period. Participants arrived at 11:00 am and were provided with a standard caloric lunch (weight and gender adjusted). At 12:00, an indwelling catheter was inserted to facilitate upcoming blood draws to assess blood alcohol and neuroendocrine response following a standard alcohol-priming dose. At $12: 30 \mathrm{pm}$, the participant received the final witnessed dose of study medication. At 2:00 pm, the curtain was pulled back to reveal the bar-like cues described above (indicating the bar 'was open') and they consumed a standard dose $(0.4 \mathrm{~g} /$ $\mathrm{kg}$ for men, $0.34 \mathrm{~g} / \mathrm{kg}$ for women) of their preferred alcoholic beverage (beer, wine, spirits), designed to achieve a blood alcohol concentration (BAC) of $50-60 \mathrm{mg} \%$. In order to improve the personal relevance of this procedure, the beverage was prepared in the fashion that they described as their preferred mode (eg on the rocks), and brand preferences were accommodated whenever possible. The beverage was consumed in the bar-like setting over a 10-min interval, with the dose split into two equal halves ( $5 \mathrm{~min}$ each). Over the next $50 \mathrm{~min}$, questionnaires related to alcohol effects and alcohol craving were administered, blood samples were taken, and participants performed a computerized reaction time task. These measures were taken to characterize differences between alcoholics and social drinkers in acute alcohol response, and to evaluate potential effects of opiate antagonist medications on these responses. Most of these data are beyond the scope of the present article and will be reported separately. At 3:00 pm (50 min after consumption of the priming alcohol drink), a 90-min alcohol choice self-administration period commenced. During this period, the participant could request up to four alcoholic 'mini-drinks,' with the alcoholic content of each drink equal to one-fourth that of the standard priming drink. The participant was told that they would receive $\$ 2$ for each drink (bar credit) that they did not consume. Money was offered as an alternative reinforcer in order to increase the motivation to abstain from drinking alcohol, thereby providing a more sensitive assay of the impact of opiate antagonist medications on the reinforcement value of alcohol. The provision of alternate reinforcers has been used in a number of studies examining the incentive value of various drugs of abuse (eg Higgins, 1997; Shahan et al, 2001). The participant was not provided with this explanation, but simply told that they could choose to drink alcohol, or receive money, or any combination of the two. The participant also had snacks and various nonalcoholic drinks available during this period.

Following the alcohol choice self-administration period, the participant was required to remain in the laboratory until 6:30 pm, or until a breathalyzer reading was below $20 \mathrm{mg} \%$, whichever time was later. Prior to dismissal, the participant was given dinner and permitted to listen to music, watch videos, or read magazines. Alcoholic participants were also given educational and self-help materials regarding alcohol effects and changing drinking behavior. Participants were either driven home by a friend or family member, or a taxi was provided. On the day following the laboratory session, the participant attended an individual feedback and debriefing session designed to increase motivation to reduce drinking or seek treatment. This semi-structured session utilized aspects of Motivational Enhancement Therapy (MET; Miller and Rollnick, 1991) and the Guided Self-Change approach (eg Sobell and Sobell, 1993). 


\section{Data Analysis}

Repeated measures analysis of variance (ANOVA) was used to examine Group (alcoholics vs social drinkers) and Medication (naltrexone vs nalmefene vs placebo) effects on blood alcohol levels achieved, with Time following the standard 'priming' drink during the bar-lab procedure used as the repeated measure. Analysis of covariance (ANCOVA) was used to examine differences in the number of drinks consumed during the initial 5-day natural environment drinking period, as well as the number of drinks consumed during the limited access alcohol consumption portion of the bar-lab session, as a function of Group and Medication status. A composite index of medication side effects (computed as the average of 22 symptoms rated at the end of the medication week) was used as a covariate for the analyses of both of these alcohol consumption variables, as there is reason to expect that side effects may influence decisions regarding whether and how much to drink. The side effect composite was not used as a covariate in the analysis of blood alcohol data, as there was no expectation that side effects would have an impact on this purely pharmacological variable. Baseline drinking (number of standard drinks consumed during the 90-day prestudy period) was used as an additional covariate in the analysis of the natural environment drinking period only. In addition, $\chi^{2}$ analysis was performed to determine if there were differences in the percentage of participants in each group choosing to consume alcohol during the limitedaccess bar-lab procedure. Finally, $\chi^{2}$ analyses were used to evaluate potential differences in reported side effects between medication groups. For these analyses, tests were first conducted to determine if there were overall differences in the percentage of participants reporting symptoms across the three medication groups, with separate analyses conducted for the alcoholic and social drinker groups. For symptoms in which a significant overall effect was found, follow-up tests were used to evaluate differences between each pair of groups (ie naltrexone $v s$ placebo, nalmefene $v s$ placebo, naltrexone $v s$ nalmefene).

\section{RESULTS}

\section{Sample Characteristics}

Table 1 shows demographic and drinking-related characteristics for social drinkers and alcoholics. There were no differences on any measures across the three medication groups for either alcoholics or social drinkers; therefore, these groups are collapsed in the present table. Overall, participants were approximately 30 years old, predominantly male, and white $(93.5 \%$ white subjects, $5.1 \%$ black subjects, $0.9 \%$ Hispanic, and $0.5 \%$ Asian). There were no significant differences between the alcoholics and social drinkers on demographic variables, nor were there differences in the proportion of participants who chose beer, wine, or liquor as their preferred beverage. However, alcoholics were more likely than social drinkers to be smokers and to have a first degree relative with a history of significant alcohol problems. Alcoholics also had significantly higher scores on all measures related to current affective disturbance and drinking involvement, as well as higher baseline levels of $\gamma$-glutamyltransferase.

\section{Medication Compliance}

We were able to estimate compliance rates by analyzing complete urine riboflavin data in $78 \%$ of the social drinkers

Table I Demographic and Drinking Characteristics

\begin{tabular}{|c|c|c|c|}
\hline & & \multicolumn{2}{|c|}{ Group } \\
\hline & & $\begin{array}{c}\text { Social drinkers } \\
(n=90)\end{array}$ & $\begin{array}{l}\text { Alcoholics } \\
(n=125)\end{array}$ \\
\hline \multicolumn{4}{|l|}{ General characteristics } \\
\hline $\begin{array}{l}\text { \% Smokers*** } \\
\text { \% Family alcohol problems*** } \\
\text { BDI*** } \\
\text { BAI*** }\end{array}$ & $\begin{array}{l}\text { \% Male } \\
\% \text { Caucasian } \\
\% \text { Beer } \\
\% \text { Wine } \\
\% \text { Liquor }\end{array}$ & $\begin{array}{l}32.0(10.9) \\
77 \\
96 \\
58 \\
18 \\
24 \\
6 \\
12 \\
1.4(2.0) \\
1.3(1.7)\end{array}$ & $\begin{array}{l}30.3(11.2) \\
82 \\
92 \\
70 \\
9 \\
21 \\
36 \\
42 \\
5.4(5.6) \\
5.2(6.2)\end{array}$ \\
\hline \multicolumn{4}{|l|}{ Drinking characteristics } \\
\hline $\begin{array}{l}\text { ADS*** } \\
\text { SAAST*** } \\
\text { OCDS** } \\
\text { ACQ*** } \\
\text { TLFB*** } \\
\text { GGT** }\end{array}$ & $\begin{array}{l}\% \text { Drinking days } \\
\text { Drinks/drinking day }\end{array}$ & $\begin{aligned} 1.3 & (1.6) \\
1.4 & (2.0) \\
2.1 & (1.2) \\
58.6 & (13.1) \\
22 & (17) \\
2.7 & (1.2) \\
29.3 & (24.2)\end{aligned}$ & $\begin{array}{r}12.3(5.4) \\
3.4(1.9) \\
12.2(4.9) \\
109.7(45.8) \\
75(20) \\
8.3(3.1) \\
55.9(84.5)\end{array}$ \\
\hline
\end{tabular}

Note: Standard deviations appear in parentheses. $\mathrm{BDI}=$ Beck Depression Inventory; $\mathrm{BAI}=$ Beck Anxiety Inventory; ADS = Alcohol Dependence Scale; SAAST = Self-Administered Alcohol Screening Test; OCDS = ObsessiveCompulsive Drinking Scale; ACQ = Alcohol Craving Questionnaire; TLFB =90-day Timeline Follow-Back; GGT $=\gamma$ glutamyltransferase. $* p<0.001$, $* * 0.0001$. 
and $82 \%$ of the alcoholics in our sample. Compliance rates were determined by calculating the percentage of participants in each group who either had a day 6 urine riboflavin level $>1500 \mathrm{ng} / \mathrm{ml}$ (see Anton et al, 1999), or a day 6 riboflavin level that was at least twice as high as the baseline (day 1) level. Estimated compliance rates in each group were as follows: social drinkers (placebo, 91\%; naltrexone, 91\%; nalmefene, 88\%), alcoholics (placebo, 89\%; naltrexone, $85 \%$; nalmefene, $87 \%$ ). Thus, overall compliance appeared to be quite good, and $\chi^{2}$ tests confirmed that compliance did not differ as a function of medication condition for social drinkers or alcoholics, $\chi^{2 \text { s }}$ [2] $<1$. It should also be noted that medication ingestion was witnessed on days 1, 6-8; therefore, data for all participants are included in the subsequent analyses.

\section{Alcohol Consumption: BAC}

Figure 1 shows BAC levels measured at 10, 25, 40, and $55 \mathrm{~min}$ for social drinker and alcoholic participants following consumption of the standard alcohol priming drink. Repeated measures ANOVA on these data indicated a significant Group difference, with alcoholics achieving higher blood alcohol levels than social drinkers, $\mathrm{F}(1,189)=22.35$, $p<0.0001$. There was also a significant effect of Time, $\mathrm{F}(3,567)=547.43, p<0.0001$, and a significant Time $\times$ Group interaction, $\mathrm{F}(3,567)=5.53, p<0.005$, suggesting that the difference between alcoholics and social drinkers was greatest at the two intermediate measurement periods. There was no main effect for Medication group assignment, and no significant Medication $\times$ Time interaction. A secondary ANOVA on these data, which included gender as an independent variable, indicated that males achieved a higher overall BAC than females, $\mathrm{F}(1,187)=14.93, p<0.001$. However, there were no gender interactions with Group, Medication, or Time (all $p$ 's $>0.29$ ).

\section{Alcohol Consumption: Natural Environment Drinking Period}

Figure 2 shows the average number of standard alcoholic beverages consumed during the first 5 days while taking

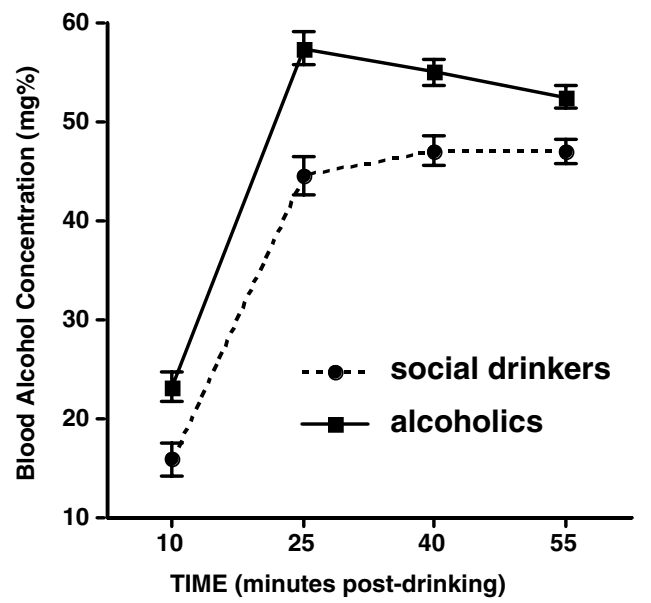

Figure I BAC ( \pm SEM) for alcoholics and social drinkers at each time point following consumption of the standard alcohol priming drink (see text for significance)

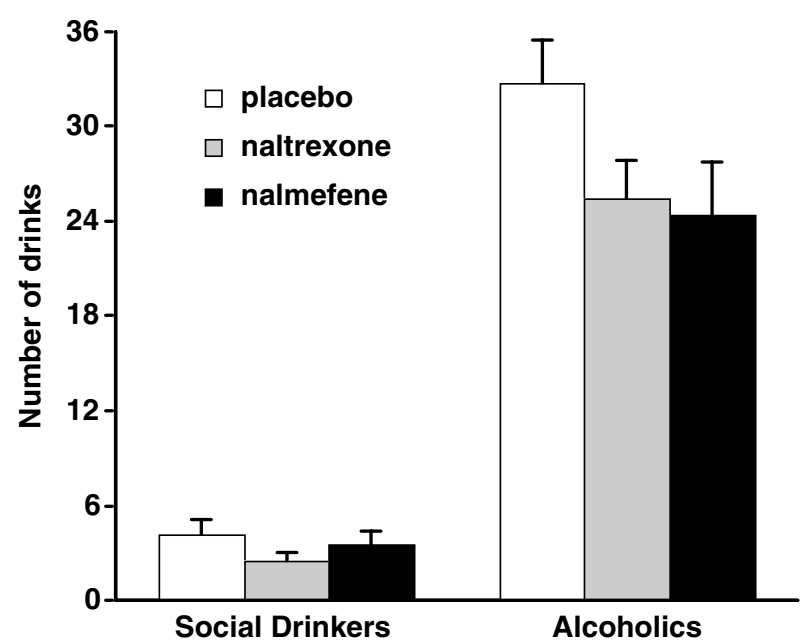

Figure 2 Number of standard drinks ( \pm SEM) consumed during the 5day medication period for alcoholics and social drinkers taking naltrexone, nalmefene, or placebo (see text for significance).

active medication or placebo. The ANCOVA on these data revealed a significant main effect for Group, $\mathrm{F}(1,192)=$ 8.63, $p<0.01$, with alcoholics drinking substantially more than the social drinkers. There was also a main effect of Medication condition, $\mathrm{F}(2,192)=3.49, p<0.05$, and a significant Group $\times$ Medication interaction, $\mathrm{F}(2,192)=3.13, p<0.05$. As far as covariates, the medication side effect composite was not significantly related to alcohol consumption during the medication week, but there was a positive association between baseline drinking and alcohol consumption during this period $(t=8.26$, $p<0.0001$, regression weight $=0.03)$. Post hoc tests indicated that there were no Medication effects on drinking among the social drinkers, but there were significant differences as a function of Medication among the alcoholics. Although the two active medication groups (naltrexone and nalmefene) did not differ from each other $(\mathrm{F}<1)$, alcoholics in both of these groups drank significantly less than alcoholics in the placebo group during this 5 -day period $(p<0.01)$.

\section{Alcohol Consumption: Limited Access Choice Self-Administration}

Figure 3 shows the proportion of participants in each group opting to consume alcohol during the limited access alcohol consumption procedure (top panel), as well as the number of drinks consumed during the procedure (bottom panel). Analyses conducted on both these variables confirmed that there were significant Group effects, with alcoholics more likely to drink $\left(\chi^{2}[1]=48.52, p<0.001\right)$ and drinking larger amounts $(F(1,184)=58.02, p<0.0001)$ than social drinkers. In addition, participants receiving active medication were less likely to drink $\left(\chi^{2}[2]=6.46, p<0.05\right)$ and drank smaller amounts $(\mathrm{F}(1,184)=4.97, p<0.05)$, relative to participants receiving placebo. There was no significant Group $\times$ Medication interaction for the drinking quantity variable $(\mathrm{F}(1,184)=1.55, p>0.2)$, suggesting that medication effects were evident for both alcoholics and social drinkers. However, planned comparisons indicated 

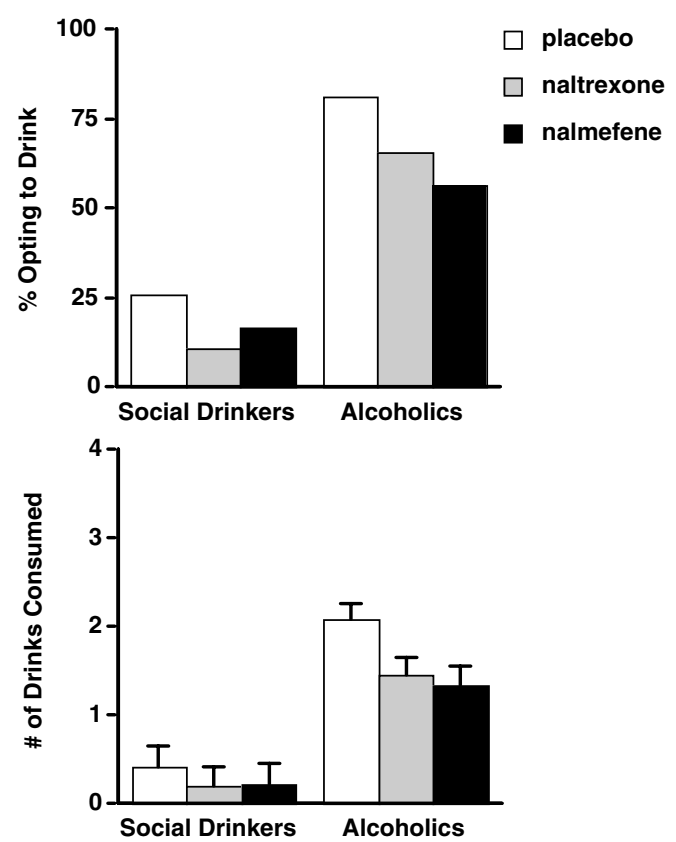

Figure 3 Percentage of participants in each medication group opting to drink during the free-drinking period (top panel), and the average number of drinks ( \pm SEM) consumed during this period (bottom panel) (see text for significance).

that naltrexone and nalmefene each reduced drinking significantly relative to placebo among alcoholics $(p$ 's $<0.05)$; these differences were not significant among social drinkers ( $p$ 's $>0.5$ ).

\section{Medication Dropouts and Side Effects}

In addition to the three early participants who were dropped from the study because of the intolerable side effects while taking the faster nalmefene titration, seven additional participants were dropped because of medication side effects. Six of these participants were taking nalmefene (three social drinkers, three alcoholics) and one was taking naltrexone (social drinker). Table 2 displays side effects that were reported at the end of the medication week (day 6) in a significantly greater proportion of either alcoholic or social drinker study completers who were taking either of the two opiate antagonist medications, relative to placebo. Four participants (two social drinkers, two alcoholics) who received the faster nalmefene titration at the beginning of the study were excluded from these analyses. Of 22 potential symptoms that were rated, eight symptoms showed significant elevations in at least one of the four active medication groups (alcoholic/naltrexone, alcoholic/nalmefene, social drinker/naltrexone, social drinker/nalmefene). It should be noted that no statistical corrections were made for multiple comparisons, so effects at the $p<0.05$ level should be interpreted cautiously. In general, nalmefene was associated with greater reporting of side effects. However, it should be further noted that the vast majority of these side effects were rated in the mild to moderate range, with very few reports of severe symptoms in the slower titration group reported here.

Table 2 Percentage of Participants Reporting Side Effects During 6 Days of Medication

\begin{tabular}{|c|c|c|c|c|}
\hline & \multicolumn{4}{|c|}{ Medication group } \\
\hline & Naltrexone & Nalmefene & Placebo & $p$ value \\
\hline \multicolumn{5}{|l|}{ Trouble sleeping } \\
\hline Alcoholics & $26^{\mathrm{a}}$ & $68^{b}$ & $32^{\mathrm{a}}$ & 0.01 \\
\hline Social drinkers & $21^{\mathrm{a}}$ & $69^{b}$ & $30^{\mathrm{a}}$ & 0.01 \\
\hline \multicolumn{5}{|l|}{ Nervousness } \\
\hline Alcoholics & $26^{\mathrm{a}}$ & $32^{\mathrm{a}}$ & $9^{b}$ & 0.05 \\
\hline Social drinkers & $11^{\mathrm{a}}$ & $31^{b}$ & $7^{\mathrm{a}}$ & 0.05 \\
\hline \multicolumn{5}{|l|}{ Irritability } \\
\hline Alcoholics & $39^{a}$ & $61^{\mathrm{b}}$ & $15^{c}$ & 0.01 \\
\hline Social drinkers & $25^{\mathrm{a}}$ & $46^{\mathrm{b}}$ & $13^{\mathrm{a}}$ & 0.05 \\
\hline \multicolumn{5}{|c|}{ Trouble Concentrating } \\
\hline Alcoholics & $18^{\mathrm{a}}$ & $45^{b}$ & $20^{\mathrm{a}}$ & 0.05 \\
\hline Social drinkers & $11^{\mathrm{a}}$ & $46^{\mathrm{b}}$ & $13^{\mathrm{a}}$ & 0.01 \\
\hline \multicolumn{5}{|l|}{ Abdominal pain } \\
\hline Social drinkers & $29^{a}$ & $23^{\mathrm{a}}$ & $3^{b}$ & 0.05 \\
\hline \multicolumn{5}{|l|}{ Nausea/vomiting } \\
\hline Alcoholics & $44^{\mathrm{a}}$ & $26^{\mathrm{b}}$ & $15^{c}$ & 0.05 \\
\hline Social drinkers & $43^{\mathrm{a}}$ & $35^{\mathrm{a}}$ & $3^{\mathrm{b}}$ & 0.01 \\
\hline \multicolumn{5}{|l|}{ Headache } \\
\hline Social drinkers & $21^{\mathrm{a}}$ & $50^{b}$ & $20^{\mathrm{a}}$ & 0.05 \\
\hline \multicolumn{5}{|l|}{$\begin{array}{l}\text { Decreased sex } \\
\text { drive/interest }\end{array}$} \\
\hline Alcoholics & $8^{\mathrm{a}}$ & $27^{\mathrm{b}}$ & $4^{\mathrm{a}}$ & 0.01 \\
\hline Social drinkers & 4 & 8 & 0 & ns \\
\hline
\end{tabular}

Note: Included in the table are symptoms in which there was a significant effect $(p<0.05)$ on the overall $\chi^{2}$ test for either the alcoholic or social drinker participants. Groups in each row that do not share superscripts differ at $p<$ 0.05 . These analyses were conducted on individuals in each medication group who completed the medication period, and did not include participants who received the faster nalmefene titration. Group sample sizes were as follows: alcoholics - 39 naltrexone, 3 I nalmefene, and 47 placebo; social drinkers - 28 naltrexone, 26 nalmefene, and 30 placebo.

\section{DISCUSSION}

The present study demonstrated drinking reductions in both a natural environment and a bar-laboratory setting among participants who were taking either of two common opiate antagonist medications, naltrexone or nalmefene. Both medications were associated with a reduced probability of drinking, as well as reduced drinking amounts. These effects were observed only in nontreatment-seeking alcoholics and not social drinkers, and were similar in direction to those observed in previous clinical treatment trials and laboratory studies.

The finding that opiate antagonist medications reduced drinking during the natural environment period among nontreatment-seeking alcoholics is particularly compelling, given that these participants were not especially motivated to reduce their drinking, nor were they instructed to do so. This effect was present even when medication side effects were controlled for in the analyses. The recent study by O'Malley et al (2002), employing a similar group of nontreatment-seeking alcoholics (albeit with somewhat lower levels of alcohol consumption), reported that naltrexone-treated participants drank approximately half of the amount of alcohol per occasion during a similar prelaboratory medication period. However, the small sample size in that study may have precluded this effect from reaching statistical significance. To our knowledge, the 
present study is the first demonstration in human alcoholics that opiate antagonist medications can reduce drinking under natural conditions in the absence of a motivated attempt to reduce or quit drinking. This is consistent with a growing literature showing effects of opiate antagonist medications on drinking in animal models (eg Froehlich et al, 1990; Middaugh and Bandy, 2000; Stromberg et al, 1998), where it is assumed that the animals have no particular desire to drink less.

Nontreatment-seeking alcoholics and social drinkers exhibited clearly different alcohol consumption patterns in the present study. Alcoholics were substantially more likely to drink and they consumed greater amounts of alcohol than social drinkers. These differences were observed both in the natural environment and in the bar-laboratory selfadministration paradigm. Furthermore, although social drinkers taking opiate antagonists showed a nonsignificant reduction in drinking during the laboratory procedure, opiate antagonist effects were only significant in the alcoholic group during this procedure and during the natural environment observation period. This suggests that opiate antagonist in nonalcoholics may not produce clinically important effects.

In contrast to the study reported by O'Malley et al (2002), but similar to Davidson et al's $(1996,1999)$ studies, participants in the present study consumed their preferred beverage (eg beer, wine, or liquor) in the bar-laboratory setting in order to add to the ecological validity of the paradigm. Despite similar types and amounts of alcohol consumed during the standard dosing period, alcoholics achieved higher peak blood alcohol levels than social drinkers in the present study. These data indicating that alcoholics achieved higher peak blood alcohol levels than social drinkers after a standard oral dose are consistent with those of others (eg Frezza et al, 1990; Lieber et al, 1994). Although chronic high alcohol induces the microsomal ethanol oxidizing system (MEOS) in the liver (Lieber, 1994), leading to faster metabolism of alcohol and other drugs in alcoholics, it has also been recognized that chronic heavy alcohol use substantially reduces first pass metabolism secondary to decreased gastric alcohol dehydrogenase activity (Frezza et al, 1990). While the MEOS effect would increase alcohol degradation in alcoholics, a diminished first pass effect in this group is consistent with the observed increased peak blood alcohol level (see Lieber, 1994). Given these differences between alcoholics and social drinkers, any interaction of medication with alcohol would appear to have less clinical relevance when studied in social drinkers only.

There were also differences in blood alcohol levels achieved by males and females, with males achieving higher overall levels. These differences were independent of medication status, drinker status, and time of assessment. It is possible that the gender adjustment in the standard dose of alcohol administered overcompensated for gender differences in body water, thus leading to higher blood alcohol levels in males. Given that the present study was not specifically powered to examine gender effects, an area for further research should be to determine whether there are differential gender effects on blood alcohol level as a function of medication status or drinking history.
We failed to find significant differences between naltrexone and nalmefene in that both medications reduced alcohol consumption to a similar degree relative to placebo. There are subtle differences in the receptor pharmacology of these compounds. Both bind strongly to $\mu$-opiate receptors in rodent (De Haven-Hudkins et al, 1990; Mitch et al, 1993), and nonhuman primate (Emmerson et al, 1994) brain cortex. However, nalmefene seems to bind more strongly to delta receptors than naltrexone when tested in rodents (De Haven-Hudkins et al, 1990; Mitch et al, 1993) and nonhuman primates (Emmerson et al, 1994). While in some functional rodent models nalmefene shows greater antagonism for $\kappa$ agonists than naltrexone (CulpepperMorgan et al, 1995), in nonhuman primates (Emmerson et $a l$, 1994) both drugs seem to have similar affinity for $\kappa$ receptors. Of interest, nalmefene, in contrast to naltrexone has been shown in human cell lines to act as an inverse agonist (ie blunt physiological function during a resting (nonstimulated) state), while naltrexone is a neutral antagonist (ie affects physiological function only after agonist stimulation) (Wang et al, 2001).

There are reports that opiate antagonists that bind to delta receptors may reduce alcohol intake as well, or better, than those that bind exclusively to $\mu$ receptors (eg Froehlich et al, 1998). The results of the present human study suggest that, if there are any differences in opiate receptor binding between naltrexone and nalmefene, these differences do not appear to differentiate clinical response, at least at the doses utilized in this study. The intolerable side effects that occurred in the initial participants who received a faster nalmefene titration ( $20 \mathrm{mg}$ on the first 2 days) might suggest that either the potency or receptor selectivity of nalmefene is different than naltrexone, as suggested previously (De Haven-Hudkins et al, 1990; Mitch et al, 1993; Emmerson et al, 1994). Alternatively, the recent characterization of nalmefene as an inverse agonist under certain conditions (Wang et al, 2001) suggests that nalmefene, in contrast to naltrexone, might cause an opiate withdrawal-like state under the right conditions. Since some of these participants complained of CNS-related effects (eg dysphoria, temperature dysregulation, cognitive disturbances, illusions, sleep difficulties) not too dissimilar to opiate withdrawal-like effects, an inverse agonist effect of nalmefene would seem plausible. This might also account for the greater incidence of mild insomnia, irritability, nervousness, and trouble concentrating reported in the nalmefene-treated participants. Although we did not systematically record the timing of the medication side effects within the medication period, they were almost universally experienced during the first few days of dosing, such that by the day of the bar-lab procedure there were minimal complaints.

When a slower titration of nalmefene was instituted in our study, these effects were not as evident. This would suggest that brain-opiate receptors could be more sensitive to a potential differential receptor binding or an inverse agonist effect of nalmefene at higher doses, but that these receptors may adapt over time. Daily ratings of adverse events could address this issue. Of note, in the clinical trial of Mason et al (1999), there were no complaints reported of this nature. In that study, while even a higher dose of nalmefene (up to $80 \mathrm{mg}$ ) was utilized, the first daily dose was $0.5 \mathrm{mg}$, which was titrated upwards over 1 week. It is 
unlikely that recent alcohol consumption played a role in our study since both alcoholics and social drinkers experienced these effects. It is noteworthy that major differences in adverse effects between naltrexone and nalmefene were mostly CNS related. Gastrointestinal effects were similar across the two medications and not unexpected given similar observations in clinical lab studies (King et al, 1997a, b; Swift et al, 1994) and clinical trials (Anton et al, 1999; Mason et al, 1994, 1999; O’Malley et al, 1992; Volpicelli et al, 1992).

The present findings need to be considered in the light of several potential study limitations. First, it is difficult to eliminate potential contamination across phases using the present study design. For instance, alcohol-related expectancies may have changed over the course of the medication week, particularly among participants who may have experienced reduced drinking or changes in alcohol-related effects because of ingestion of an active opiate antagonist medication (eg O'Malley et al, 2002; Swift et al, 1994). Numerous studies have shown that alcohol-related expectances can impact later alcohol consumption (see Goldman, 2002). It should be noted that the present study was not meant to necessarily isolate opiate antagonist effects to one particular phase of the study. Rather, our intent was to evaluate these effects within a paradigm that balanced experimental control with a resemblance of how these medications are typically used in clinical treatment settings. Indeed, it may be that expectancy change is a critical factor within the pharmacological treatment of alcoholism. This issue might be addressed more explicitly in future research by allowing one group of participants to drink $a d$ lib during the medication week, while holding alcohol consumption among another group constant, perhaps by requiring complete abstinence. This manipulation was considered beyond the scope of the present research, yet may be important for further mechanistic work in this area. In the present study, it was intended that the standard 2-day abstinence period would, to some extent, establish a common base immediately prior to the bar-lab session.

Another limitation concerns the alcoholic sample recruited for this study. As the present participants were all nontreatment seekers, they tended to be somewhat younger and had less extensive and less severe alcohol involvement than typical treatment samples. Nonetheless, their drinking patterns were diagnostically relevant, and clearly excessive relative to the social drinkers. At the very least, this population of nontreatment-seeking alcoholics offers distinct advantages as compared with studying strictly social drinker groups, in that they are more closely aligned with the types of alcoholics who are most likely to benefit from the medications being evaluated. It may be feasible in the future to employ similar paradigms with alcoholics exhibiting even more chronic and severe alcohol problems. However, more studies with nontreatment seekers should first be conducted in order to establish the validity and safety of the procedures. Related to this point, a brief telephone assessment was conducted 6 weeks after the present laboratory session with alcoholic participants to assess drinking during the period subsequent to the lab session. Reported findings from this assessment suggest a positive impact of participating in this protocol on drinking behavior, in that alcoholics report meaningful decreases in the frequency and intensity of their alcohol consumption following study participation (Drobes and Anton, 2000). This suggests that participation of nontreatment-seeking alcoholics has minimal negative effects on alcohol consumption and, in fact, has mostly positive effects as measured by reduction in drinking. Whether this is because of participation in the protocol or a result of the guided selfchange feedback is not certain.

In summary, the present clinical laboratory paradigm appears to provide a reliable procedure to evaluate medication effects on alcohol consumption in a relevant population of alcoholics. The finding that naltrexone and nalmefene reduced alcohol intake in both natural environment and bar-lab settings suggests that the paradigm may work well for testing other medications that could be clinically useful. Finally, we previously reported that participation in this paradigm by nontreatment-seeking alcoholics does not exacerbate alcohol consumption after participation (Drobes and Anton, 2000), so similar studies may be conducted with alcoholics in the future with the confidence that they are ethical and benign.

\section{ACKNOWLEDGEMENTS}

This study was conducted at the Medical University of South Carolina under the auspices of the Charleston Alcohol Research Center, funded by NIAAA Grant P50 AA10761. The authors express appreciation to Suzanne Wise, Heather Brown, and Catherine Kelly for their valuable help in data collection, and to Wei Wang for statistical analyses. We also thank Dupont (naltrexone) and IVAX/ Baker Norton (nalmefene) for donating the medications and matching placebos used in this study. Reprint requests and correspondence can be directed to either David J Drobes, who is now at the H Lee Moffitt Cancer Center \& Research Institute, University of South Florida, 4115 E. Fowler Ave., Tampa, FL 33617 (e-mail: drobesdj@moffitt.usf.edu) or Raymond F Anton, Charleston Alcohol Research Center, Medical University of South Carolina, 67 President St., PO Box 250861, Charleston, SC 29425 (e-mail: antonr@musc. edu).

\section{REFERENCES}

American Psychiatric Association (1994). Diagnostic and Statistical Manual of Mental Disorders, 4th edn. American Psychiatric Association: Washington, DC.

Andreasen NC, Endicott J, Spitzer RL, Winokur G (1977). The family history method using diagnostic criteria. Arch Gen Psychiatry 34: 1229-1235.

Anton RF, Moak DH, Latham P (1995). The obsessive compulsive drinking scale: a self-rated instrument for the quantification of thoughts about alcohol and drinking behavior. Alcohol Clin Exp Res 19: 92-99.

Anton RF, Moak DH, Waid LR, Latham PK, Malcolm RJ, Dias JK (1999). Naltrexone and cognitive behavioral therapy for the treatment of outpatient alcoholics: results of a placebocontrolled trial. Am J Psychiatry 156: 1758-1764.

Beck AT (1967). Depression: Clinical, Experimental, and Theoretical Aspects. Hoeber: New York.

Beck AT, Epstein N, Brown G, Steer RA (1988). An inventory for measuring clinical anxiety: psychometric properties. J Consult Clin Psychol 56: 893-897. 
Chick J, Anton R, Checinski K, Croop R, Drummond DC, Farmer R et al (2000). A multicentre, randomized, double-blind, placebocontrolled trial of naltrexone in the treatment of alcohol dependence or abuse. Alcohol Alcohol 35: 587-593.

Culpepper-Morgan JA, Holt PR, LaRoche D, Kreek MJ (1995). Orally administered opioid antagonists reverse both mu and kappa opioid agonist delay of gastrointestinal transit in the guinea pig. Life Sci 56: 1187-1192.

Davidson D, Palfai T, Bird C, Swift R (1999). Effects of naltrexone on alcohol self-administration in heavy drinkers. Alcohol Clin Exp Res 23: 195-203.

Davidson D, Swift R, Fitz E (1996). Naltrexone increases the latency to drink alcohol in social drinkers. Alcohol Clin Exp Res 20: 732-739.

Davis LJ, Hurt RD, Morse RM, O’Brien PC (1987). Discriminant analysis of the Self-Administered Alcoholism Screening Test. Alcohol Clin Exp Res 11: 269-273.

De Haven-Hudkins DL, Brostrom PA, Allen JT, Lesko LJ, Ferkany JW, Kaplita PV et al (1990). Pharmacologic profile of NPC168 (naltrexonephenyl oxime), a novel compound with activity at opioid receptors. Pharmacol Biochem Behav 37: 497-504.

de Wit H, Svenson J, York A (1999). Non-specific effect of naltrexone on ethanol consumption in social drinkers. Psychopharmacology 146: 33-41.

Drobes DJ, Anton RF (2000). Drinking in alcoholics following an alcohol challenge research protocol. J Stud Alcohol 61: 220-224.

Emmerson PJ, Liu M, Woods JH, Medzihradsky F (1994). Binding affinity and selectivity of opioids at mu, delta, and kappa receptors in monkey brain membranes. J Pharmacol Exp Therap 271: 1630-1637.

First MB, Spitzer RL, Gibbon M, Williams JBW (1994). Structured clinical interview for axis I DSM-IV disorders - patient edition (SCID-I/P, version 2.0). Biometrics Research Department, New York State Psychiatric Institute.

Frezza M, Di Padova C, Pozzato G, Terpin M, Baraona E, Lieber CS (1990). High blood alcohol levels in women: the role of decreased gastric alcohol dehydrogenase activity and first-pass metabolism. $N$ Engl J Med 322: 95-99.

Froehlich JC, Badia-Elder NE, Zink RW, McCullough DE, Portoghese PS (1998). Contribution of the opioid system to alcohol aversion and alcohol drinking behavior.J Pharmacol Exp Ther 287: 284-292.

Froehlich JC, Harts J, Lumeng L, Li TK (1990). Naloxone attenuates voluntary alcohol intake in rats selectively bred for high alcohol preference. Pharmacol Biochem Behav 35: 385-390.

Goldman MA (2002). Expectancy and risk for alcoholism: the unfortunate exploitation of a fundamental characteristic of neurobehavioral adaptation. Alcohol Clin Exp Res 26: 737-746.

Higgins ST (1997). The influence of alternative reinforcers on cocaine use and abuse: a brief review. Pharmacol Biochem Behav 57: 419-427.

King AC, Volpicelli JR, Frazer A, O’Brien CP (1997a). Effect of naltrexone on subjective alcohol response in subjects at high and low risk for future alcohol dependence. Psychopharmacology 129: $15-22$.

King AC, Volpicelli JR, Gunduz M, O’Brien CP, Kreek MJ (1997b). Naltrexone biotransformation and incidence of subjective side effects: a preliminary study. Alcohol Clin Exp Res 21: 906-909.

Kranzler HR, Van Kirk J (2001). Efficacy of naltrexone and acamprosate for alcoholism treatment: a meta-analysis. Alcohol Clin Exp Res 25: 1335-1341.

Krystal JH, Cramer JA, Krol WF, Kirk GF, Rosenheck RA (2001). Naltrexone in the treatment of alcohol dependence. $N$ Engl J Med 345: 1734-1739.

Lieber CS (1994). Hepatic and metabolic effects of ethanol: pathogenesis and prevention. Ann Med 26: 325-330.
Lieber CS, Gentry RT, Baraona E (1994). First pass metabolism of ethanol. Alcohol Alcohol 2(Suppl): 163-169.

Mason BJ, Ritvo EC, Morgan RO, Salvato FR, Goldberg G, Welch B et al (1994). A double-blind, placebo-controlled pilot study to evaluate the efficacy and safety of oral nalmefene HCL for alcohol dependence. Alcohol Clin Exp Res 18: 1162-1167.

Mason BJ, Salvato FR, Williams LD, Ritvo EC, Cutler RB (1999). A double-blind, placebo-controlled study of oral nalmefene for alcohol dependence. Arch Gen Psychiatry 56: 719-724.

McCaul ME, Wand GS, Rohde C, Lee SM (2000). Serum 6-betanaltrexol levels are related to alcohol responses in heavy drinkers. Alcohol Clin Exp Res 24: 1385-1391.

Middaugh LD, Bandy AE (2000). Naltrexone effects on ethanol consumption and response to ethanol conditioned cues in C57BL/6 mice. Psychopharmacology 151: 321-327.

Miller WR, Rollnick S (1991). Motivational Interviewing: Preparing People to Change Addictive Behavior. Guilford Press: New York.

Mitch CH, Leander JD, Mendelsohn LG, Shaw WN, Wong DT, Cantrell BE et al (1993). 3,4-dimethyl-4-(3-hydroxyphenyl) piperidines: opioid antagonists with potent anorectant activity. J Med Chem 36: 2842-2850.

National Advisory Council on Alcohol Abuse and Alcoholism (1988). Recommended council guidelines on ethyl alcohol administration in human experimentation. US DHHS, ADAMHA: Rockville, MD.

O’Malley S, Croop R, Wroblewski J, Labriola D, Volpicelli J (1995). Naltrexone in the treatment of alcohol dependence: a combined analysis of two trials. Psychiatric Ann 25: 11-25.

O'Malley SS, Jaffe AJ, Chang G, Schottenfeld RS, Meyer RE, Rounsaville B (1992). Naltrexone and coping skills therapy for alcohol dependence: a controlled study. Arch Gen Psychiatry 49: 881-887.

O’Malley SS, Krishnan-Sarin S, Farren C, Sinha R, Kreek MJ (2002). Naltrexone decreases craving and alcohol self-administration in alcohol-dependent subjects and activates the hypothalamicpituitary-adrenocortical axis. Psychopharmacology 160: 19-29.

Palfai T, Davidson D, Swift R (1999). Influence of naltrexone on cue-elicited craving among hazardous drinkers: the moderational role of positive outcome expectancies. Exp Clin Psychopharmacol 7: 266-273.

Shahan TA, Bickel WK, Badger GJ, Giordano LA (2001). Sensitivity of nicotine-containing and de-nicotinized cigarette consumption to alternative non-drug reinforcement: a behavioral economic analysis. Behav Pharmacol 12: 277-284.

Singleton EG, Tiffany ST, Henningfield JE (1999). Alcohol craving questionnaire (ACQ-NOW): background, scoring, and administration (Revised). Intramural Research Program NIDA: Baltimore, MD.

Skinner HA, Allen BA (1982). Alcohol dependence syndrome: measurement and validation. J Abnorm Psychol 91: 199-209.

Sobell LC, Sobell MB (1992). Timeline follow-back: a technique for assessing self-reported alcohol consumption. In: Allen J, Litten RZ (eds). Measuring Alcohol Consumption: Psychosocial and Biological Methods. Humana Press: Totowa, NJ. pp 41-72.

Sobell MB, Sobell LC (1993). Problem drinkers: guided self-change treatment. Guilford Press: New York.

Stout RL, Wirtz PW, Carbonari JP, Del Boca FK (1994). Ensuring balanced distribution of prognostic factors in treatment outcome research. J Stud Alcohol 12(Suppl): 70-75.

Stromberg MF, Volpicelli JR, O'Brien CP (1998). Effects of naltrexone administered repeatedly across 30 or 60 days on ethanol consumption using a limited access procedure in the rat. Alcohol Clin Exp Res 22: 2186-2191.

Sullivan JT, Naranjo CA, Sellers EM (1989a). Compliance among heavy alcohol users in clinical drug trials. J Subst Abuse 1: 183194.

Sullivan JT, Sykora K, Schneiderman J, Naranjo CA, Sellers EM (1989b). Assessment of alcohol withdrawal: the revised clinical 
institute withdrawal assessment of alcohol scale (CIWA-ar). $\mathrm{Br} J$ Addict 84: 1353-1357.

Swift RM, Whelihan W, Kuznetsov O, Buongiorno G, Hsuing $\mathrm{H}$ (1994). Naltrexone-induced alterations in human ethanol intoxication. Am J Psychiatry 151: 1463-1467.

Volpicelli JR, Alterman AI, Hayashida M, O’Brien CP (1992). Naltrexone in the treatment of alcohol dependence. Arch Gen Psychiatry 49: 876-880.

Volpicelli JR, Rhines KC, Rhines JS, Volpicelli LA, Alterman AI, O'Brien CP (1997). Naltrexone and alcohol dependence: role of subject compliance. Arch Gen Psychiatry 54: 737-742.

Wang D, Raehal KM, Bilsky EJ, Sadee W (2001). Inverse agonists and neutral antagonists at $\mu$ opioid receptor (MOR): possible role of basal receptor signaling in narcotic dependence. $J$ Neurochem 77: 1590-1600. 